\title{
A Comparison between Linear and Nonlinear Forecasts for Nonlinear AR Models
}

\author{
MEIHUI GUO* AND Y. K. TSENG \\ National Sun Yat-sen University, Taiwan, ROC
}

\begin{abstract}
In this paper the relative forecast performance of nonlinear models to linear models is assessed by the conditional probability that the absolute forecast error of the nonlinear forecast is smaller than that of the linear forecast. The comparison probability is explicitly expressed and is shown to be an increasing function of the distance between nonlinear and linear forecasts under certain conditions. This expression of the comparison probability may not only be useful in determining the predictor, which is either a more accurate or a simpler forecast, to be used but also provides a good explanation for an odd phenomenon discussed by Pemberton. The relative forecast performance of a nonlinear model to a linear model is demonstrated to be sensitive to its forecast origins. A new forecast is thus proposed to improve the relative forecast performance of nonlinear models based on forecast origins. (C) 1997 John Wiley \& Sons, Ltd.
\end{abstract}

J. forecast, Vol. 16, 491-508 (1997)

No. of Figures: 13. No. of Tables: 1. No. of References: 8.

KEY WORDS forecast origin; multi-step forecast; SETAR model

\section{INTRODUCTION}

Prediction is an important topic in time series analysis. Many stationary phenomena in practice can be described or at least be approximated by stationary linear time series models, e.g. the $\operatorname{ARMA}(p, q)$ models (see, for example, Priestly, 1989). However, many nonlinear phenomena such as limit cycles, frequency modulations (Tong, 1990) and animal population cycles (Oster and Ipaktchi, 1978) cannot be described adequately by linear time series models, unless superfluous parameters are involved with. Intuitively, if the true model is a nonlinear time series model, then any statistical inferences using analysis for the nonlinear model, which capture the nonlinear characteristic of the data, should be better than those by using linear approximation.

\footnotetext{
* Correspondence to: Meihui Guo, Department of Applied Mathematics, National Sun Yat-sen University, Kaohsiung, ROC 80424, Taiwan.
}

Contract grant sponsor: National Science Council of the Republic of China; Contract grant number: NSC 82-0208-N$110-064$ 
However, this is not always the case for the prediction problem. For example, Davies et al. (1988) and Pemberton (1989), by numerical simulation, observe the phenomenon that the conditional mean and conditional median forecasts of nonlinear time series models have poor forecast performance compared to those of linear models. Therefore, if the linear forecast is not very much worse than the nonlinear forecast, then it is reasonable to adopt the former forecast rather than the nonlinear forecast, at least for computational reasons. More importantly, in practical applications for a given data set, unless an additional test is done in advance, there is no way of knowing whether the true model is factually linear or nonlinear. Thus, there arises the question: Under what circumstances should the nonlinear predictors be adopted?

The present research is motivated by Davies et al. (1988) who conducted three experiments to compare the forecast performance of nonlinear SETAR (Self-Exciting Threshold AutoRegressive) models with linear AR models. In experiment I they carried out the identification and estimation of SETAR models for real time series data sets. In experiments II and III they simulated series for different lengths respectively from each of 50 randomly selected stationary $\operatorname{SETAR}(2 ; 1,1)$ models of the form

$$
X_{n}=\left\{\begin{array}{lll}
a X_{n-1}+\varepsilon_{n} & \text { if } \quad X_{n-1} \leqslant 0 \\
b X_{n-1}+\varepsilon_{n} & \text { if } \quad X_{n-1}>0
\end{array}\right.
$$

where $\varepsilon_{n}$ 's are i.i.d. $N(0,1)$ r.v's. For each set of the data, both nonlinear SETAR models and linear AR models were fitted. Among three experiments the relative frequencies, of the events that the absolute forecast error of an $m$-step $(m=1,2,3)$ nonlinear forecast is smaller than that of the linear AR forecast, range from $48 \%$ to $69 \%$ when the conditional mean is used as the forecast and range from $47 \%$ to $71 \%$ when the conditional median is used. These results strongly imply that there is a positive probability that the linear forecast is better than the nonlinear forecast even when the true model is nonlinear.

Pemberton (1989) conducted simulations for model (1) with $a=-b=0.4$ and 0.9 and fitted generated data by AR models. For the conditional median forecast, the conditional probability that the absolute forecast error of the $m$-step nonlinear forecast is smaller than that of the AR forecast given $\vec{X}_{t}=\left(X_{1}, \ldots, X_{t}\right)$, is $P_{m}\left(\vec{X}_{t}\right)=\max \left\{F_{m}\left(Q_{m}\left(\vec{X}_{t}\right) \mid \vec{X}_{t}\right), 1-F_{m}\left(Q_{m}\left(\vec{X}_{t}\right) \mid \vec{X}_{t}\right)\right\}$, where $F_{m}\left(\cdot \mid \vec{X}_{t}\right)$ is the conditional distribution function of $X_{t+m}$ given $\vec{X}_{t}$ and $Q_{m}\left(\vec{X}_{t}\right)$ is the midpoint of the two forecasts. It can be shown that $P_{m}\left(\vec{X}_{t}\right)$ is not less than $1 / 2$. Pemberton, by plotting $P_{m}\left(\vec{X}_{t}\right)$ against the values of the stationary density function of the true model, observed that the nonlinear conditional median forecast performs better at those forecast origin points whose probability density has low values than at those points whose probability density has high ones (in the sense that $P_{m}\left(\vec{X}_{t}\right)$ is far greater than or close to 0.5 ). Pemberton also conducted his simulation for the simple piecewise constant model:

$$
X_{n}=\left\{\begin{array}{rll}
\alpha+\varepsilon_{n} & \text { if } & X_{n-1} \leqslant 0 \\
-\alpha+\varepsilon_{n} & \text { if } & X_{n-1}>0
\end{array}\right.
$$

with $\alpha=3$ and fitted the data with a linear AR model by least squares method. Pemberton observed that (1) for lags $m=1,2, \ldots, 30$, the minima of $P_{m}\left(\vec{X}_{t}\right)$, RMSE and RMAE appear in a vicinity of the modes of the stationary distribution. The RMSE (RMAE) denotes the Ratio of the Mean Squared (Absolute) Error of the conditional mean (median, correspondingly) forecast by using the nonlinear model to that by using the AR model; (2) when $m \rightarrow \infty$, RMSE (decreasingly) and RMAE (not necessarily decreasingly) tend to one whereas $P_{m}\left(\vec{X}_{t}\right)$ tends to $1 / 2$. 
Recently, Tiao and Tsay (1994) compare the post-sample forecast of TAR model versus linear AR(2) model for US quarterly real GNP data by the ratio of mean squared forecast errors. The nonlinear TAR model only outperforms the linear AR model by a substantial margin in shortterm forecasts at two regimes of the four forecast regimes.

All the above examples show that for the prediction problem, the nonlinear forecast may not be more accurate than the linear forecast, even when the true model is nonlinear and that both linear and nonlinear forecasts are asymptotically equivalent for long-term prediction. In order to better understand the poor forecast problem of the nonlinear time series models, the properties of the forecast performance criterion $P_{m}\left(\vec{X}_{t}\right)$ considered by both Davies et al. and Pemberton are studied in this paper. Under certain conditions, the criterion $P_{m}\left(\vec{X}_{t}\right)$ is shown to be an increasing function of the distance between linear and nonlinear forecasts. This result gives a nice explanation for the odd phenomena discussed by Pemberton (1989). In their simulation studies, since the distance between two forecasts are minimized around the modes of the stationary distribution of the process, the criterion $P_{m}\left(\vec{X}_{t}\right)$ is minimized at forecast regions of high probability density (see Example 1 below). For time series forecasting, usually either conditional means or conditional medians are used as optimal forecasts. However, from the above-mentioned studies and as will also be shown in this paper, the relative forecast performance (for both conditional mean and conditional median) of a nonlinear model to a linear model is rather sensitive to the forecast origins. Given a nonlinear time series, one can theoretically find a linear model which well approximates the true nonlinear models.

The organization of the paper is as follows. In the next section definitions and symbols are presented. In the third section the relation between $P_{m}\left(\vec{X}_{t}\right)$ and the distance between linear and nonlinear forecasts is exposed. An example is given to illustrate the odd phenomenon discussed by Pemberton. In the fourth section a new method is suggested which selects forecasts from conditional means or conditional medians by comparing their forecast performance with linear models. An example of piecewise constant SETAR model is also given. General conclusions will be given in the fifth section. All the technical proofs are given in the Appendix.

\section{DEFINITIONS AND NOTATIONS}

Consider the nonlinear autoregressive model of order $p(\operatorname{NLAR}(p))$

$$
X_{n}=\lambda_{0}\left(X_{n-1}, \ldots, X_{n-p}\right)+\varepsilon_{n}
$$

where $\lambda_{0}(\cdot)$ is a measurable function from $\mathbf{R}^{p}$ to $\mathbf{R}, \varepsilon_{n}$ 's are i.i.d. r.v's with mean zero, variance $\sigma^{2}$ and a common probability density function $g(\cdot)$. Model (3) includes linear, SETAR and exponentially autoregressive (see Ozaki, 1985) models as special cases. Throughout this paper, the process $\left\{X_{n}\right\}$ defined by model (3) is assumed to be stationary. Assuming the observations $\vec{X}_{t}=\left(X_{1}, X_{2}, \ldots, X_{t}\right)$ (capitals are used for both random variables and realizations) is generated from model (3), the unknown value of $X_{t+m}(m \geqslant 1)$ will be predicted by either the conditional mean or the conditional median when $\vec{X}_{t}$ is given. Since the conditional mean $E\left(X_{t+m} \mid \vec{X}_{t}\right)$ minimizes the mean squared error, it is also called the MMSE forecast. Similarly, the conditional median of $X_{t+m}$ when $\vec{X}_{t}$ is given will be called the MMAE (Minimum Mean Absolute Error) forecast. In general, there are no closed forms of the MMSE and MMAE forecasts for $\operatorname{NLAR}(p)$ models, except some numerical algorithms that may be available. The 
goal of this paper is to compare the $\operatorname{NLAR}(p)$ forecast with the linear $\operatorname{AR}(p)$ forecast when the true model is factually $\operatorname{NLAR}(p)$. Define

$$
P_{m}\left(\vec{X}_{t}\right)=P\left(\left|X_{t+m}-N L_{m}\left(\vec{X}_{t}\right)\right|<\mid X_{t+m}-A R_{m}\left(X_{t}\right) \|, \vec{X}_{t}\right)
$$

where $N L_{m}\left(\vec{X}_{t}\right)$ is the nonlinear $m$-step MMSE or MMAE forecast and $A R_{m}\left(\vec{X}_{t}\right)$ is the linear $\operatorname{AR}(p) m$-step conditional mean forecast. When $N L_{m}\left(\vec{X}_{t}\right) \neq A R_{m}\left(\vec{X}_{t}\right)$, it can be shown that

$$
P_{m}\left(\vec{X}_{t}\right)=F_{m}\left(Q_{m}\left(\vec{X}_{t}\right) \mid \vec{X}_{t}\right) I_{\left[R^{-}\right]}+\left(1-F_{m}\left(Q_{m}\left(\vec{X}_{t}\right) \mid \vec{X}_{t}\right) I_{\left[R^{+}\right]}\right.
$$

where $F_{m}\left(\cdot \mid \vec{X}_{t}\right)$ is the conditional distribution of $X_{t+m}$ given $\vec{X}_{t}, Q_{m}\left(\vec{X}_{t}\right)=\frac{1}{2}\left(N L_{m}\left(\vec{X}_{t}\right)+\right.$ $\left.A R_{m}\left(\vec{X}_{t}\right)\right), \quad I_{[\cdot]}$ is the indicator function, $R^{-}=\left\{\vec{X}_{t}: N L_{m}\left(\vec{X}_{t}\right)<A R_{m}\left(\vec{X}_{t}\right)\right\}$, and $R^{+}=$ $\left\{\vec{X}_{t}: N L_{m}\left(\vec{X}_{t}\right)>A R_{m}\left(\vec{X}_{t}\right)\right\}$. The probability $P_{m}\left(\vec{X}_{t}\right)$ is considered as a criterion to compare the forecast performance, by Davies et al. as well as by Pemberton. Finally, we denote $\xi_{m}\left(\vec{X}_{t}\right)=\left|N L_{m}\left(\vec{X}_{t}\right)-A R_{m}\left(\vec{X}_{t}\right)\right|$, the distance between the nonlinear and linear forecasts at the forecast origin $\vec{X}_{t}$.

\section{FORECAST ACCURACY AND FORECAST DISTANCE}

In this section the comparison probability $P_{m}\left(\vec{X}_{t}\right)$ and the ratio of mean squared forecast errors are shown to be increasing functions of the forecast distance $\xi_{m}\left(\vec{X}_{t}\right)$ (see Theorems 1 and 5 below). Throughout this paper, $\mu_{m}\left(\vec{X}_{t}\right)$ denotes the $m$-step conditional mean and $M_{m}\left(\vec{X}_{t}\right)$ the $m$-step conditional median of the $\operatorname{NLAR}(p)$ model.

Theorem 1 Suppose that $\left(X_{1}, X_{2}, \ldots, X_{t}\right)$ is observed from the NLAR( $\left.p\right)$ model (3). If we write $F_{m}\left(x \mid \vec{X}_{t}\right)=F\left(x-N L_{m}\left(\vec{X}_{t}\right)\right)$, then

$$
P_{m}\left(\vec{X}_{t}\right)=F\left(\frac{1}{2} \xi_{m}\left(\vec{X}_{t}\right)\right) I_{\left[R^{-}\right]}+\left(1-F\left(-\frac{1}{2} \xi_{m}\left(\vec{X}_{t}\right)\right)\right) I_{\left[R^{+}\right]}
$$

This equation shows that for the MMAE forecast, $P_{m}\left(\vec{X}_{t}\right) \geqslant 1 / 2$ a.s., if $P\left(\xi_{m}\left(\vec{X}_{t}\right)=0\right)=0$. If the distribution $F(\cdot)$ is continuous and symmetric about 0 , then $P_{m}\left(\vec{X}_{t}\right)=F\left(\frac{1}{2} \xi_{m}\left(\vec{X}_{t}\right)\right)$.

Corollary 2 Let $G(\cdot)$ denote the distribution function of the innovations. For multi-step forecast (i.e. $m>1$ ), the distribution function $F$ in Theorem 1 is given by $F(x)=$ $E\left[G\left(x-\lambda_{0}\left(X_{t+m-1}, \ldots, X_{t+m-p}\right)+N L_{m}\left(\vec{X}_{t}\right)\right) \mid \vec{X}_{t}\right]$. Especially for the one-step MMSE forecast, since $N L_{1}\left(\vec{X}_{t}\right)=\lambda_{0}\left(X_{t}, \ldots, X_{t+1-p}\right)$, we have $P_{1}\left(\vec{X}_{t}\right)=G\left(\frac{1}{2} \xi_{1}\left(\vec{X}_{t}\right)\right) I_{\left[R^{-}\right]}+\left(1-G\left(-\frac{1}{2} \xi_{1}\left(\vec{X}_{t}\right)\right)\right) I_{\left[R^{+}\right]}$.

The proofs of Theorem 1 and Corollary 2 are immediate and hence omitted.

Note that $P_{m}\left(\vec{X}_{t}\right)$ is the probability that the nonlinear forecast is better than the linear forecast. Therefore, we may say that the nonlinear forecast is better than the linear forecast if $P_{m}\left(\vec{X}_{t}\right)>1 / 2$. Theorem 1 shows that the nonlinear MMAE forecast is not worse than the linear MMAE forecast. However, it should be noted that this conclusion is not true practically since the theoretic nonlinear forecast $N L_{m}\left(\vec{X}_{t}\right)$ is factually unknown in real situations. In real applications, the nonlinear AR function $\lambda_{0}\left(X_{t+m-1}, \ldots, X_{t+m-p}\right)$ is usually unknown and only its estimates are available. Even if it were known, the forecast $N L_{m}\left(\vec{X}_{t}\right)$ cannot be exactly computed and only 
an approximation of it may be available. This shows that the nonlinear forecast may not be actually better than the linear forecast.

For the MMSE forecasts, the nonlinear forecast may not be better than the linear forecast, even theoretically. For example, when the distribution of the innovations satisfies $G\left(\frac{1}{2} \xi_{1}\left(\vec{X}_{t}\right)\right)<1 / 2$ and the origin is in the set $R^{-}$; or $1-G\left(-\frac{1}{2} \xi_{1}\left(\vec{X}_{t}\right)\right)<1 / 2$ and the origin is in the set $R^{+}$. If the error in the computation of the MMSE nonlinear forecast is taken into account, the MMSE nonlinear forecast will further lose its superiority to the linear forecast.

If the regressor $\lambda_{0}$ has an explicit form and the distribution of the innovations is known, sometimes the conditional distribution $F_{m}\left(x \mid \vec{X}_{t}\right)$ of $X_{t+m}$ can be explicitly expressed. As an example, consider the piecewise constant SETAR model:

$$
X_{n}=\alpha_{j}+\varepsilon_{n} \quad \text { if } \quad X_{n-1} \in\left(c_{j-1}, c_{j}\right]
$$

where $j=1,2, \ldots, l,-\infty=c_{0}<c_{1}<\cdots<c_{l}=\infty, \varepsilon_{n}$ 's are i.i.d. $N(0,1)$ random variables.

Theorem 3 Consider the $m$-step MMSE forecast when the true model is (7). In this case, we have $N L_{m}\left(\vec{X}_{t}\right)=\mu_{m}\left(\vec{X}_{t}\right)$. When $X_{t} \in\left(c_{j-1}, c_{j}\right], j=1,2, \ldots, l$, we have

$$
P_{m}\left(\vec{X}_{t}\right)=P_{m}\left(X_{t}\right)=H_{m, j}\left(\frac{\xi_{m}\left(X_{t}\right)}{2}\right) I_{\left[R^{-}\right]}+\left(1-H_{m, j}\left(-\frac{\xi_{m}\left(X_{t}\right)}{2}\right)\right) I_{\left[R^{+}\right]}
$$

where $H_{m, j}(x)=\sum_{i=1}^{l} k_{i j}^{(m)} \Phi\left(x+\alpha_{i j}^{(m)}\right), \forall j=1,2, \ldots, l, k_{i j}^{(m)}$ are elements of the matrix $K^{m-1}$ with $K=\left(\Phi\left(c_{i}-\alpha_{j}\right)-\Phi\left(c_{i-1}-\alpha_{j}\right)\right)$ and $\alpha_{i j}^{(m)}=-\alpha_{i}+\sum_{i=1}^{l} k_{i j}^{(m)} \alpha_{1}$.

Corollary 4 For model (2), $N L_{m}\left(\vec{X}_{t}\right)=\mu_{m}\left(\vec{X}_{t}\right)=-\alpha \beta^{m-1} \operatorname{sign}\left(X_{t}\right)$, the comparison probability has the following explicit form:

$$
P_{m}\left(X_{t}\right)=H_{m}\left(\frac{\xi_{m}\left(X_{t}\right)}{2}\right) I_{\left[R^{-}\right]}+\left(1-H_{m}\left(-\frac{\xi_{m}\left(X_{t}\right)}{2}\right)\right) I_{\left[R^{+}\right]}
$$

where $H_{m}(x)=k_{1}^{(m)} \Phi\left(x-2 \operatorname{sign}\left(X_{t}\right) \alpha k_{2}^{(m)}\right)+k_{2}^{(m)} \Phi\left(x+2 \operatorname{sign}\left(X_{t}\right) \alpha k_{1}^{(m)}\right), \quad k_{1}^{(m)}=\left(1-\beta^{m-1}\right) / 2$, $k_{2}^{(m)}=\left(1+\beta^{m-1}\right) / 2$, and $\beta=1-2 \Phi(\alpha)$.

Now, consider the ratio of the mean squared (absolute) errors of the nonlinear forecasts to linear forecasts under model (3). Define

$$
\operatorname{RMSE}_{m}\left(\vec{X}_{t}\right)=\frac{\left[\left(X_{t+m}-A R_{m}\left(\vec{X}_{t}\right)\right)^{2} \mid \vec{X}_{t}\right]}{\operatorname{MSE}\left(\mu_{m}\left(\vec{X}_{t}\right) \mid \vec{X}_{t}\right)}
$$

where

$$
\begin{aligned}
\operatorname{MSE}\left(\mu_{m}\left(\vec{X}_{t}\right) \mid \vec{X}_{t}\right) & =E\left[\left(X_{t+m}-\mu_{m}\left(\vec{X}_{t}\right)\right)^{2} \mid \vec{X}_{t}\right] \\
& =\sigma^{2}+E\left[\left(\lambda_{0}\left(X_{t+m-1}, \ldots, X_{t+m-p}\right)-\mu_{m}\left(\vec{X}_{t}\right)\right)^{2} \mid \vec{X}_{t}\right]
\end{aligned}
$$

and define

$$
R M A E_{m}\left(\vec{X}_{t}\right)=\frac{E\left[\mid\left(X_{t+m}-A R_{m}\left(\vec{X}_{t}\right) \| \vec{X}_{t}\right]\right.}{\operatorname{MAE}\left(M_{m}\left(\vec{X}_{t}\right) \mid \vec{X}_{t}\right)}
$$

where $\operatorname{MAE}\left(M_{m}\left(\vec{X}_{t}\right) \mid \vec{X}_{t}\right)=E\left[\mid\left(X_{t+m}-M_{m}\left(\vec{X}_{t}\right) \| \vec{X}_{t}\right]\right.$. 
The following theorem shows that both $\operatorname{RMSE}_{m}\left(\vec{X}_{t}\right)$ and $\operatorname{RMAE}\left(\vec{X}_{t}\right)$ are increasing functions of $\xi_{m}\left(\vec{X}_{t}\right)$.

Theorem 5 If model (3) is the true model, then

$$
\operatorname{RMSE}_{m}\left(\vec{X}_{t}\right)=1+\frac{\left(\xi_{m}\left(\vec{X}_{t}\right)\right)^{2}}{\operatorname{MSE}\left(\mu_{m}\left(\vec{X}_{t}\right) \mid \vec{X}_{t}\right)}
$$

and

$$
R M A E_{m}\left(\vec{X}_{t}\right)=1+\frac{2\left|\int_{M_{m}\left(\vec{X}_{t}\right)}^{A R_{m}\left(\vec{X}_{t}\right)}\left(x-A R_{m}\left(\vec{X}_{t}\right)\right) \mathrm{d} F_{m}\left(x \mid \vec{X}_{t}\right)\right|}{\operatorname{MAE}\left(M_{m}\left(\vec{X}_{t}\right) \mid \vec{X}_{t}\right)}
$$

Next, let us consider the $m$-step forecast for large $m$ when model (3) is the true model. We say that the time series $\left\{X_{t}\right\}$ defined by model (3) is causal if for all $t, X_{t}$ can be written as a function of $\varepsilon_{t}, \varepsilon_{t-1}, \ldots$. In this case, we have $\sigma\left(X_{t}, X_{t-1}, \ldots\right) \subset \sigma\left(\varepsilon_{t}, \varepsilon_{t-1}, \ldots\right)$. When $\left\{X_{t}\right\}$ defined by model (3) is stationary and causal, we have

$$
\mu_{m}\left(\vec{X}_{t}\right)=E\left(X_{t+m} \mid \vec{X}_{t}\right) \stackrel{\mathcal{D}}{=} E\left(X_{0} \mid B^{-t-m} \vec{X}_{t}\right) \in \sigma\left(\varepsilon_{-m}, \varepsilon_{-m-1}, \ldots\right)
$$

where $B$ is the backward shift operator, that is, $B X_{t}=X_{t-1}$. By Kolmogorov's zero-one law, $\mu_{m}\left(\vec{X}_{t}\right)$ tends to a constant as $m \rightarrow \infty$. It is easy to see that the limit of $\mu_{m}\left(\vec{X}_{t}\right)$ is the mean of $X_{t}$. Similarly, we can show that $M_{m}\left(\vec{X}_{t}\right)$ tends to the median of $X_{t}$ and that $A R_{m}\left(\vec{X}_{t}\right)$ tends to the mean of $X_{t}$. Thus, we proved the following theorem.

Theorem 6 In addition to the condition of Theorem 1, we assume that the time series $\left\{X_{t}\right\}$ is stationary and causal. When $m$ is large, for the conditional mean nonlinear forecast, we have

$$
\begin{aligned}
P_{m}\left(\vec{X}_{t}\right) \approx & F(0) I_{\left[R^{-}\right]}+(1-F(0)) I_{\left[R^{+}\right]} \\
& R M S E_{m}\left(\vec{X}_{t}\right) \rightarrow 1
\end{aligned}
$$

and for the conditional median nonlinear forecast,

$$
\begin{gathered}
P_{m}\left(\vec{X}_{t}\right) \rightarrow \begin{cases}F\left(\frac{1}{2} \mid \text { mean }- \text { medium } \mid\right) & \text { if median }<\text { mean } \\
=\frac{1}{2} & \text { if median }=\text { mean } \\
1-F\left(-\frac{1}{2} \mid \text { mean }- \text { median } \mid\right) & \text { if median }>\text { mean }\end{cases} \\
R M A E_{m}\left(\vec{X}_{t}\right) \rightarrow 1+\frac{2 \mid \int_{\text {median }}^{\text {mean }}(x-\text { mean }) \mathrm{d} F_{m}\left(x \mid \vec{X}_{t}\right)}{E \mid X_{t}-\text { median } \mid}
\end{gathered}
$$

Note that the $F(0)$ in model (8) may not be $1 / 2$ since $F$ is centerized at the mean, whereas $F(0)=1 / 2$ in model (9) since $F$ is centerized at the median.

In the following, we give an example to illustrate the results in this section. 
Example 1 Suppose that $\vec{X}_{t}=\left(X_{1}, \ldots, X_{t}\right)$ is observed from model (2) and an AR(1) model is fitted to the data. The autoregressive coefficient $\phi$ is estimated by the least squares estimate

$$
\hat{\phi}=\frac{\sum_{i=2}^{t} X_{i} X_{i-1}}{\sum_{i=1}^{t} X_{i}^{2}}
$$

The linear conditional mean forecast is $A R_{m}\left(X_{t}\right)=\hat{\phi}^{m} X_{t}$ and the nonlinear forecast is $\mu_{m}\left(X_{t}\right)=-\alpha \beta^{m-1} \operatorname{sign}\left(X_{t}\right)$, where $\beta=1-2 \Phi(\alpha)$. The conditional distribution of $X_{t+m}$ given $\vec{X}_{t}$ is $F_{m}\left(x \mid \vec{X}_{t}\right)=\frac{1}{2}\left[\Phi(x+\alpha)\left(1+\beta^{m-1} \operatorname{sign}\left(X_{t}\right)\right)+\Phi(x-\alpha)\left(1-\beta^{m-1} \operatorname{sign}\left(X_{t}\right)\right)\right]$. From this, the conditional median forecast $M_{m}\left(\vec{X}_{t}\right)$ can be evaluated. Figures 1 and 2 give the plots of $\mu_{m}\left(X_{t}\right)$, $M_{m}\left(\vec{X}_{t}\right)$ and $A R_{m}\left(X_{t}\right)$ versus $X_{t}$, with $\alpha=1.5$. The forecast distance of two conditional means is $\xi_{m}\left(X_{t}\right)=\left|\hat{\phi}^{m} X_{t}+\alpha \beta^{m-1} \operatorname{sign}\left(X_{t}\right)\right|$. Note that the least squares estimate $\hat{\phi}$ converges to

$$
\rho=\frac{E\left(X_{1} X_{2}\right)}{E\left(X_{1}^{2}\right)}=-\frac{\alpha(2 \varphi(\alpha)-\alpha \beta)}{1+\alpha^{2}}
$$

as $t \rightarrow \infty$, where $\varphi$ is the density function of the standard normal distribution. If $\alpha=1.5$, then $\hat{\phi} \cong-0.7$. Figure 3 is the plot of $\xi_{m}\left(X_{t}\right)$ versus $X_{t}$, Figures $4-6$ the plots of $P_{m}\left(X_{t}\right)$ (given by

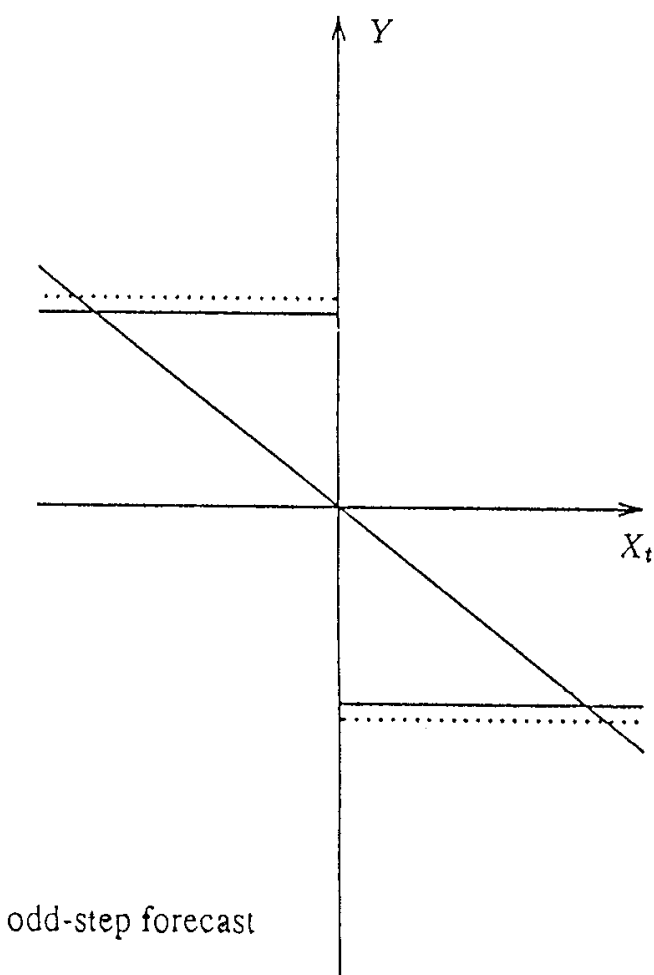

Figure 1. Conditional mean and conditional median forecasts versus origins $X_{1}$ for the AR(1) model and model (2) with $\alpha=1.5$ : odd-step forecast. $\longrightarrow$ Conditional mean, $\cdots \cdots$ conditional median

(C) 1997 John Wiley \& Sons, Ltd.

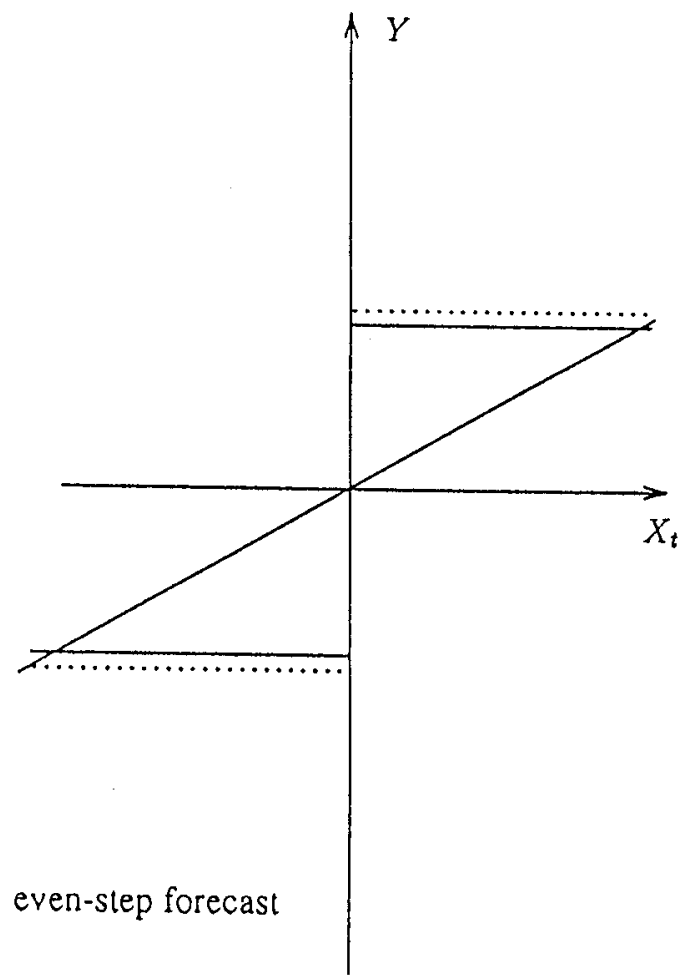

Figure 2. Even-step forecast

J. forecast, Vol. 16, 491-508 (1997) 


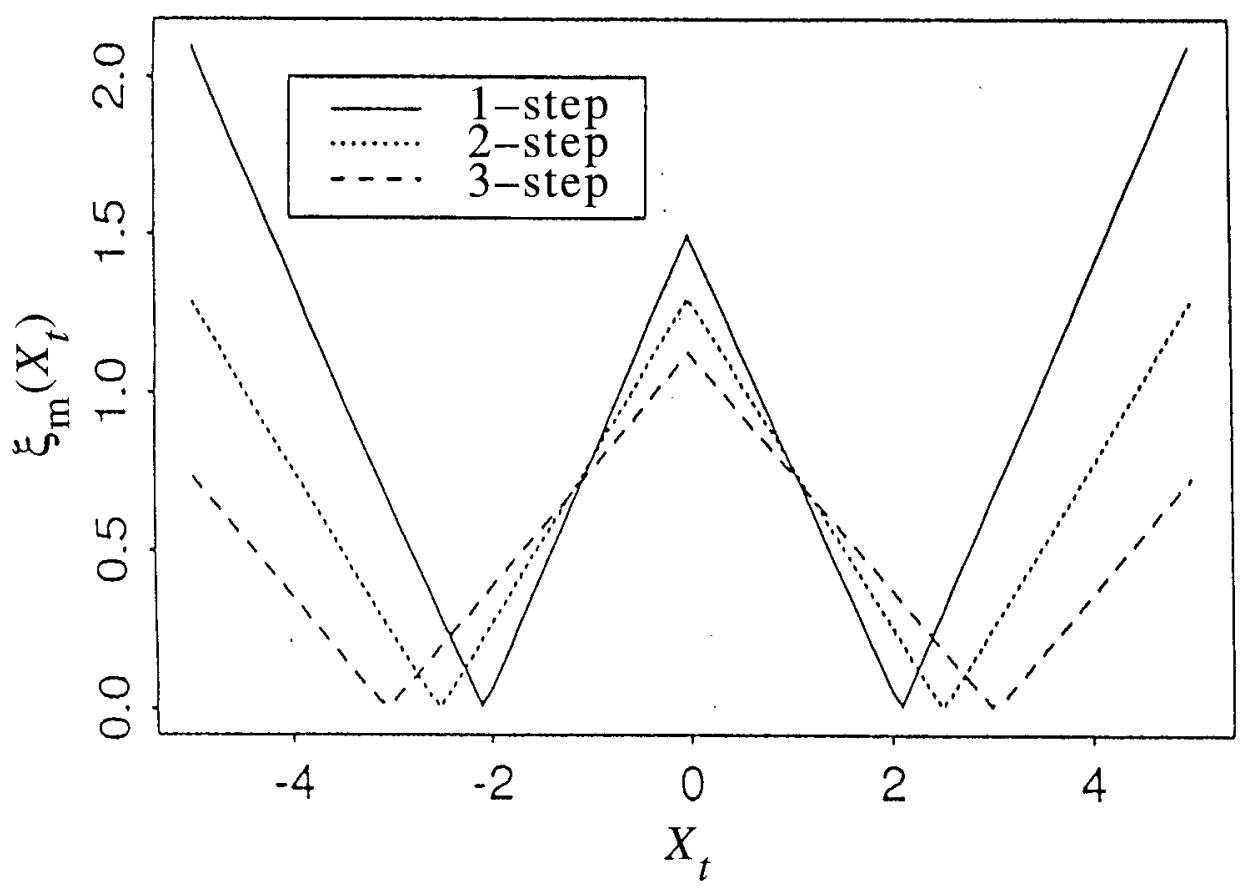

Figure 3

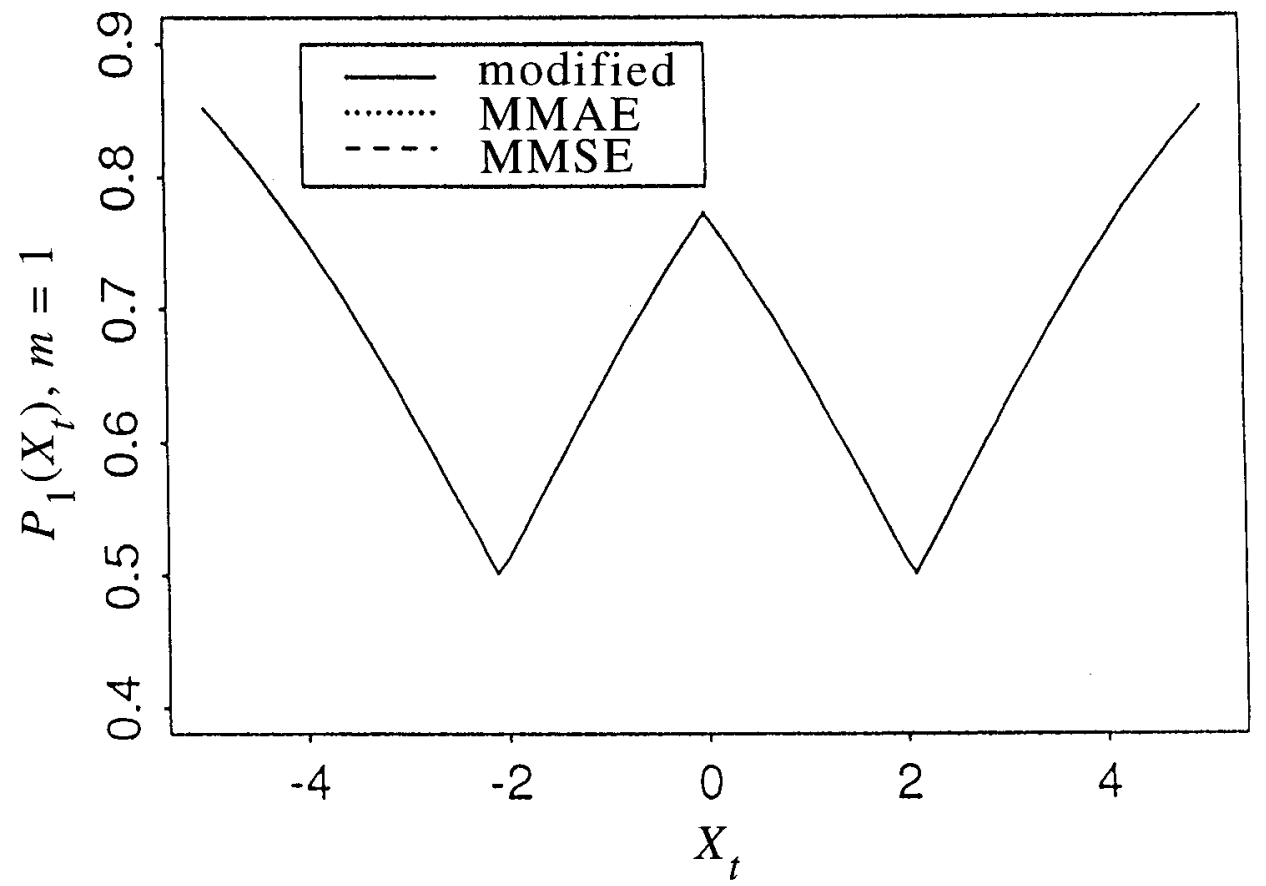

Figure 4 


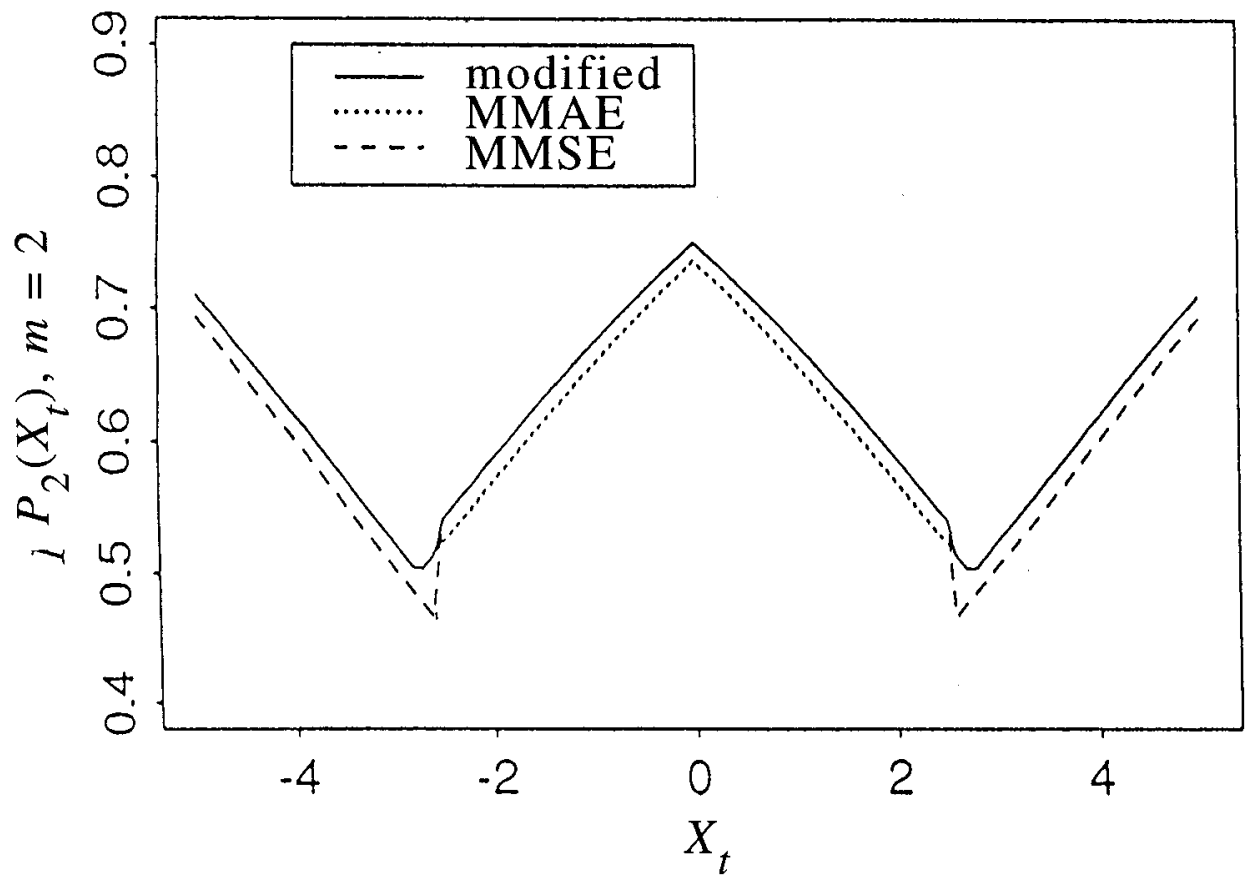

Figure 5

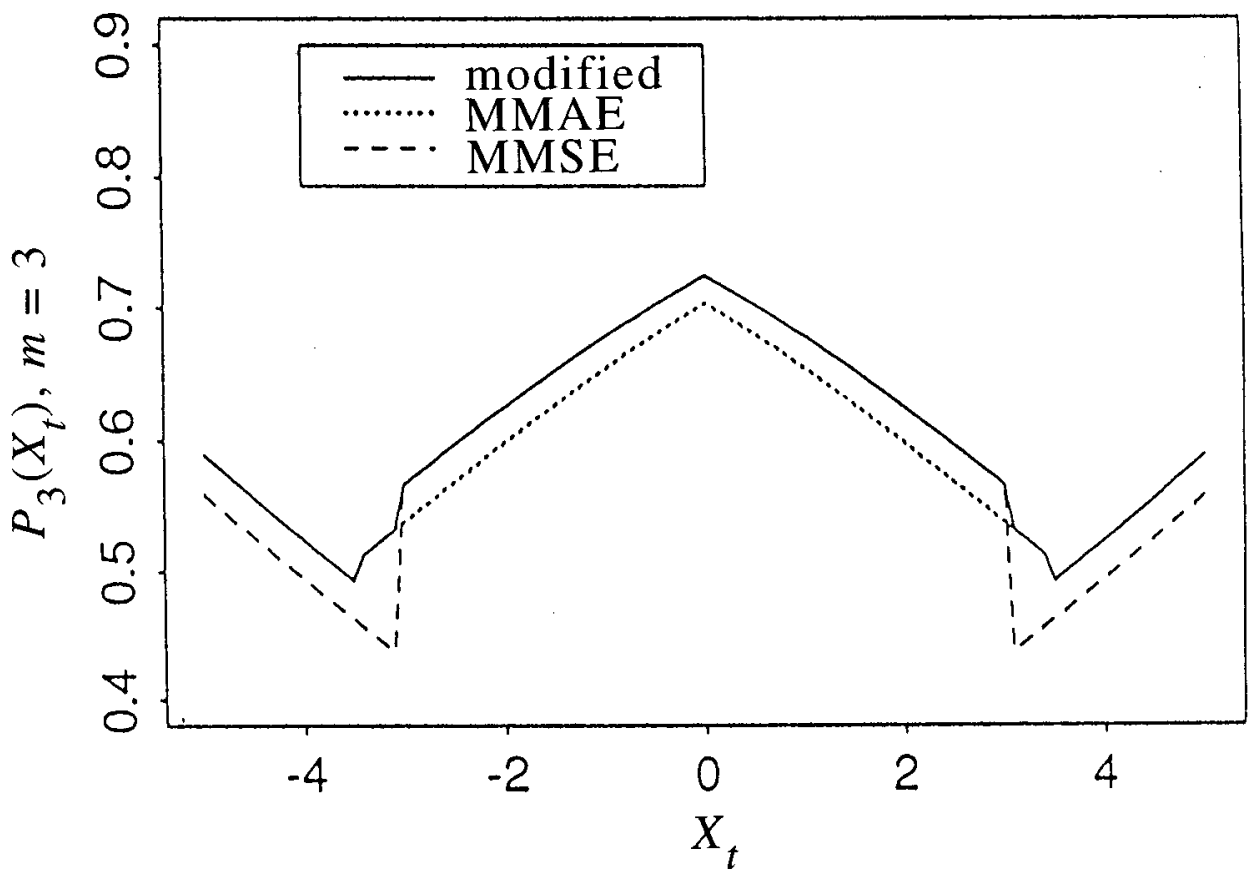

Figure 6

(C) 1997 John Wiley \& Sons, Ltd. 


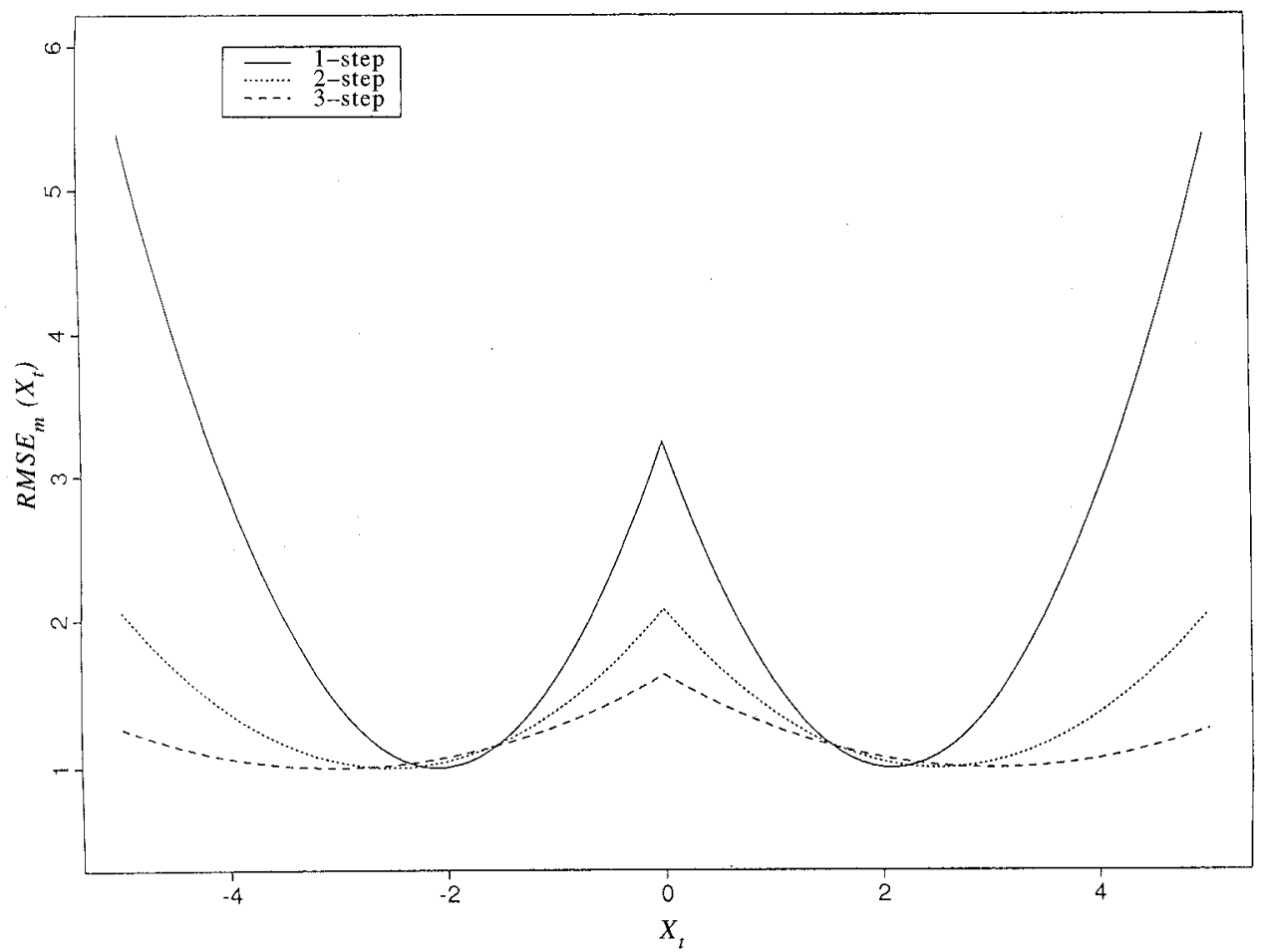

Figure 7. $R M S E_{m}\left(X_{t}\right)$ versus $X_{t}$ for the AR(1) model and model (2) with $\alpha=1.5$

Corollary 4) versus $X_{t}$ (the definition of the modified forecast is given in Theorem 7) and Figure 7 the plot of $R M S E_{m}\left(X_{t}\right)$ versus $X_{t}$, where

$$
R M S E_{m}\left(X_{t}\right)=1+\frac{\left(\xi_{m}\left(X_{t}\right)\right)^{2}}{1+\alpha^{2}\left(1-\beta^{2 m-2}\right)}
$$

Clearly, from Figure 7 it can be seen that the $\operatorname{RMSE}_{m}\left(X_{t}\right)$ is not necessarily a decreasing function in $m$ at all origins $X_{t}$. This is contrary to Pemberton's observation about $\operatorname{RMSE}_{m}\left(\vec{X}_{t}\right)$ as mentioned earlier. When $t$ is large, the intersection of the two $m$-step conditional means given $X_{t}$ (by equating the two conditional means) is given by

$$
\left|X_{t}\right|=\frac{-\alpha \beta^{m-1}}{\hat{\varphi}^{m}} \approx\left|\frac{\alpha}{\beta}\left[\frac{\alpha^{2}+1}{\alpha^{2}+\Delta(\alpha)}\right]^{m}\right| \rightarrow \infty
$$

as $m \rightarrow \infty$ where

$$
\Delta(\alpha)=-\frac{2 \alpha \varphi(\alpha)}{\beta} \in(0,1)
$$

When $\alpha=1.5$, the stationary density

$$
f(x)=\frac{\varphi(x+\alpha)+\varphi(x-\alpha)}{2}
$$

J. forecast, Vol. 16, 491-508 (1997)

(C) 1997 John Wiley \& Sons, Ltd. 
Table I. The intersection points of model (2) with $\alpha=1.5$ and AR(1) model when $X_{t} \leqslant 0$

\begin{tabular}{lcccccccccc}
\hline Step $m$ & 1 & 2 & 3 & 4 & 5 & 6 & 7 & 8 & 9 & 10 \\
\hline$-\alpha \beta^{m-1} / \hat{\phi}^{m}$ & 2.08 & 2.51 & 3.02 & 3.64 & 4.38 & 5.28 & 6.36 & 7.66 & 9.23 & 11.12 \\
\hline
\end{tabular}

of model (2) has modes around $x= \pm 1.5$ and the intersection points for $x_{t} \leqslant 0$ are given in Table I. Since the intersection points of the two forecasts are not in the neighbourhood of the modes of the stationary density function, contrary to Pemberton's observation (see above), the poor forecast region is not in the region of high density. See Figures 8-11 for the plots of $P_{m}\left(\vec{X}_{t}\right)$ of $\mu_{m}\left(\vec{X}_{n t}\right)$ versus the stationary density of $X_{t}$. However, if $\alpha$ is large but $m$ is not, then $\beta^{m-1} / \hat{\phi}^{m} \approx 1$ and $\alpha \beta^{m-1} / \hat{\phi}^{m} \approx \alpha$. For example, in Pemberton's experiment $\alpha=3$, the intersection points are close to the modes $X_{t}= \pm 3$ of the stationary p.d.f. Thus, Pemberton observe a minimum of $P_{m}\left(X_{t}\right)$ and $R M S E_{m}\left(X_{t}\right)$ in the vicinity of the modes when $m$ is small. Furthermore, since for all $X_{t}, \xi_{m}\left(X_{t}\right) \rightarrow 0$ as $m \rightarrow \infty$, thus $P_{m}\left(X_{t}\right) \rightarrow 1 / 2$ and $\operatorname{RMSE}_{m}\left(X_{t}\right) \rightarrow 1$ as $m \rightarrow \infty$, which implies the multi-step forecast performance of linear model is asymptotically as good as that of the true nonlinear piecewise constant model.

\section{A NEWLY PROPOSED FORECAST}

Calculating the nonlinear conditional mean forecast, in general, is much easier than computing the conditional median forecast. However, the former is sometimes worse than the AR predictor whose computation is the easiest among the three. If one wants to use the nonlinear forecast for

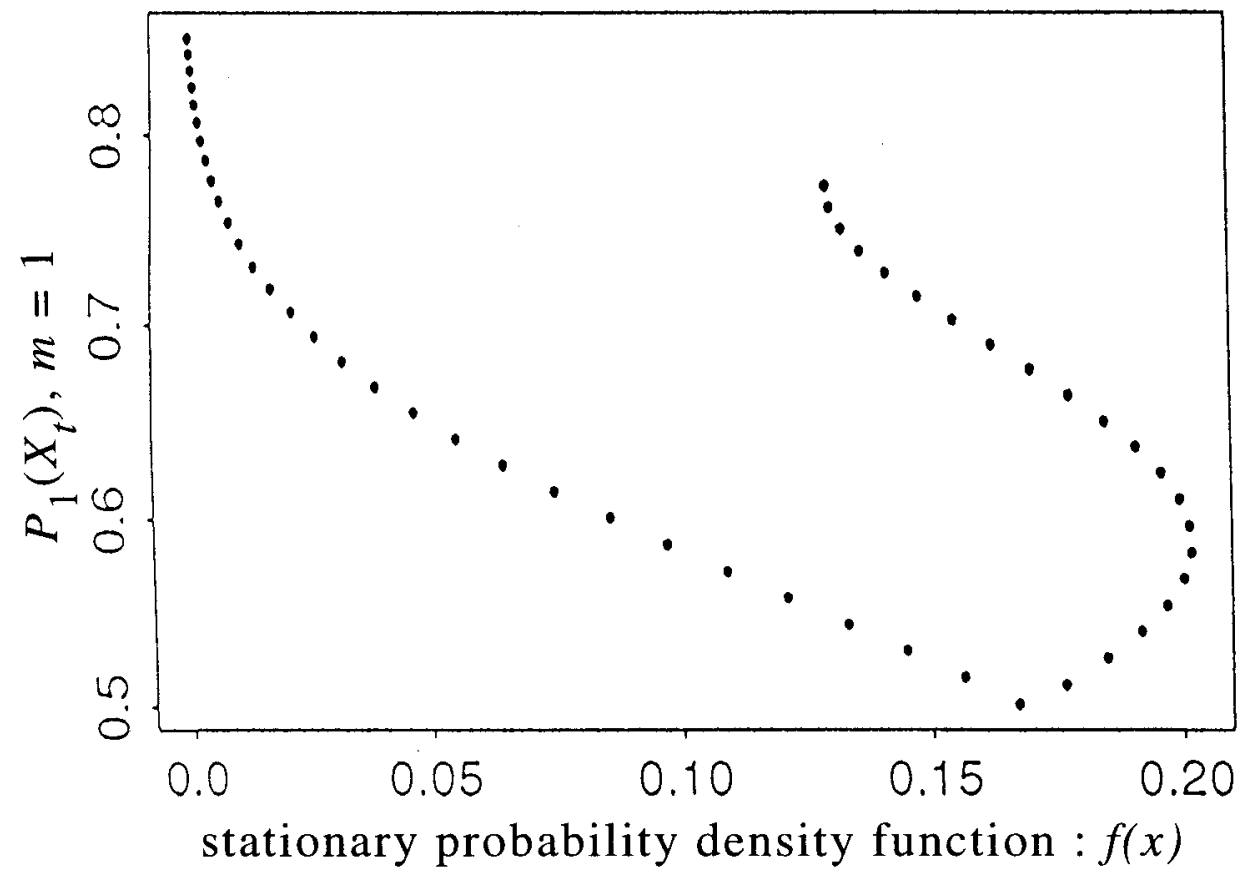

Figure 8

(C) 1997 John Wiley \& Sons, Ltd.

J. forecast, Vol. 16, 491-508 (1997) 


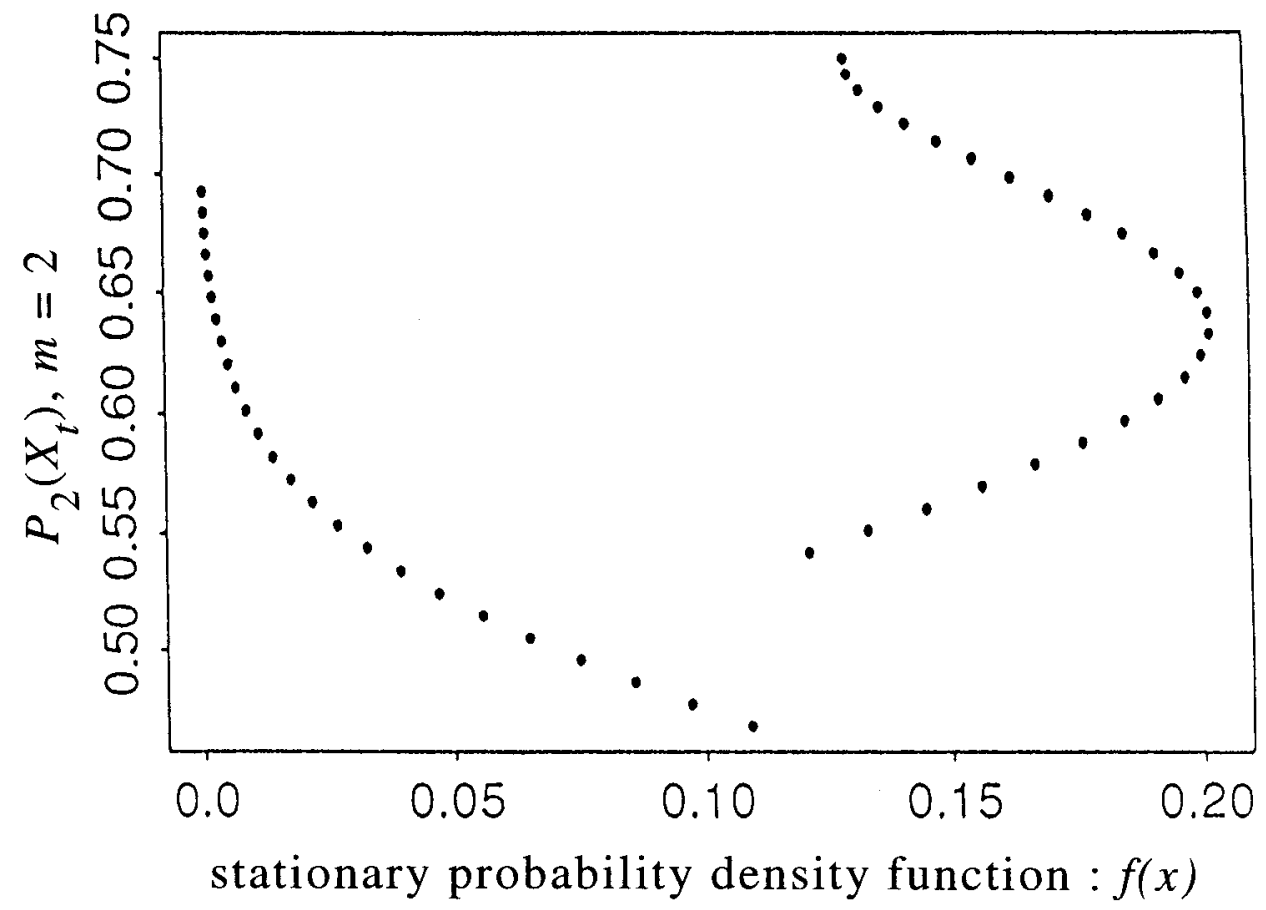

Figure 9

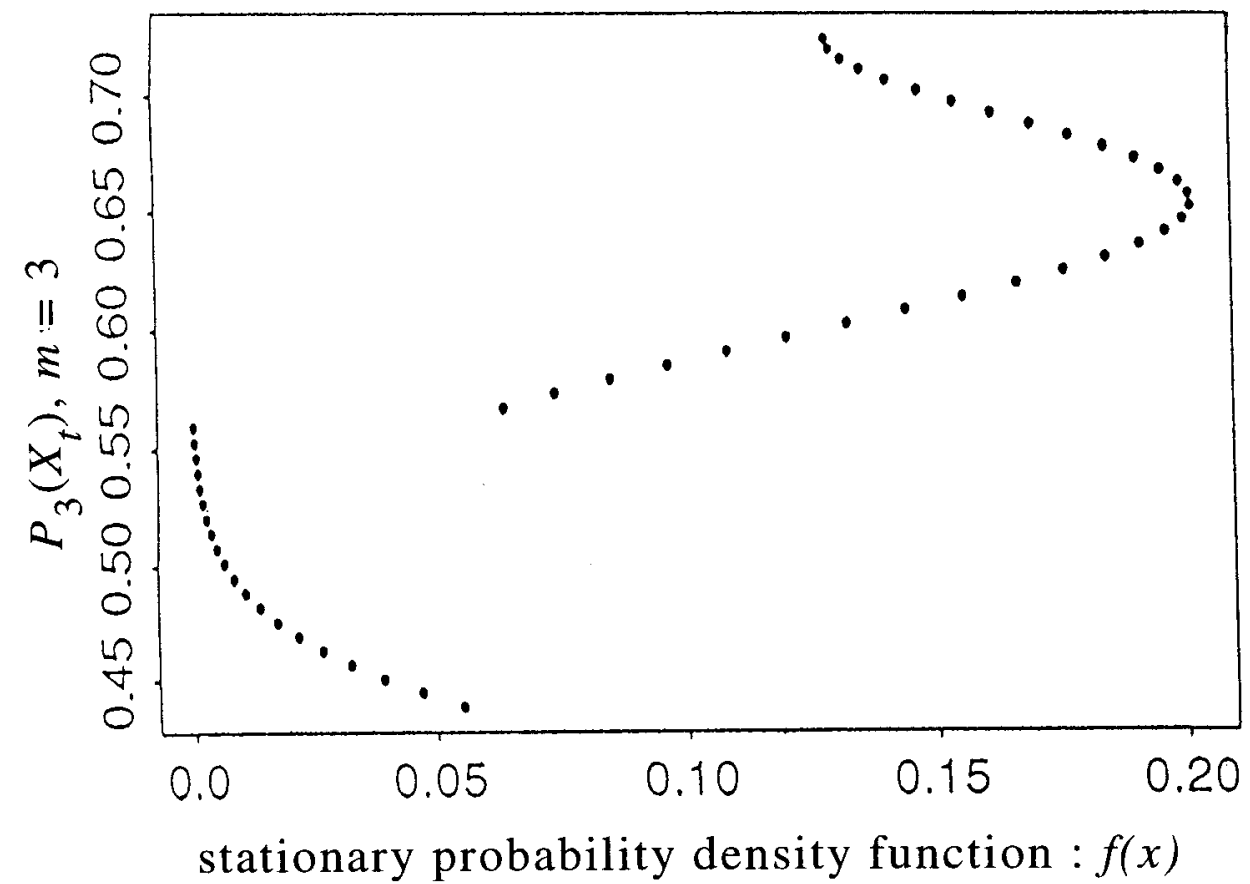

Figure 10 


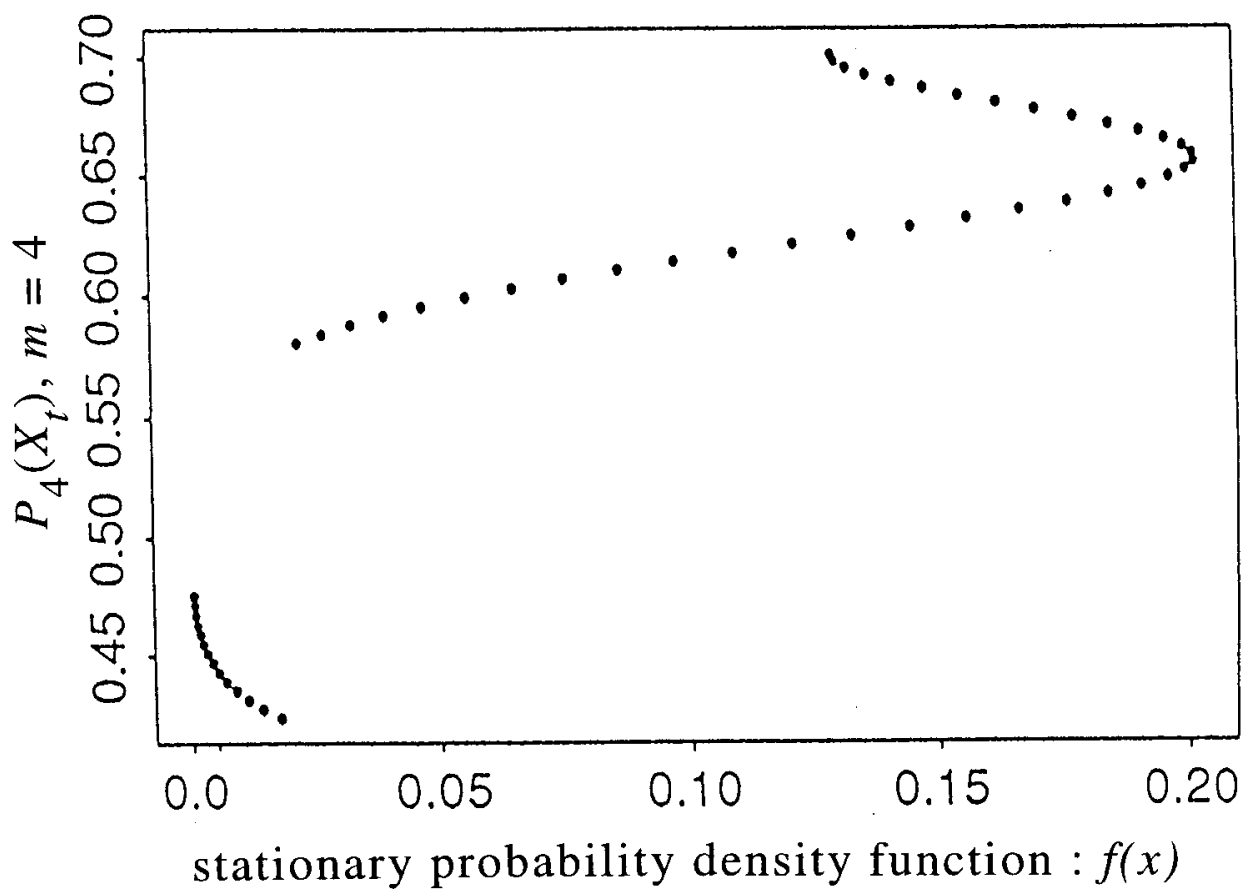

Figure 11

not losing the benefits of the nonlinear modelling, we suggest a new forecast which has the highest comparison probability relative to the AR forecast.

Consider the case when the true model is a nonlinear one. Then the $m$-step conditional probability distribution function of $X_{t+m}$ given $\vec{X}_{t}$ is usually skewed. Thus, the MMSE forecast is liable to be influenced by the skewness of the conditional distribution; furthermore, its $P_{m}\left(\vec{X}_{t}\right)$ is sometimes less than 0.5 . For the MMAE forecast, although its comparison probability $P_{m}\left(\vec{X}_{t}\right)$ is always greater than or equal to 0.5 , yet its value is more difficult to compute. Therefore, we suggest an alternative forecast, combining the MMSE forecast, and the MMAE forecast, which has a higher comparison probability $P_{m}\left(\vec{X}_{t}\right)$. The proposed forecast $M F_{m}\left(\vec{X}_{t}\right)$ is defined as

$$
\begin{array}{rlrlr}
M F_{m}\left(\vec{X}_{t}\right) & =\mu_{m}\left(\vec{X}_{t}\right) I_{\left[R^{-}\right]}+M_{m}\left(\vec{X}_{t}\right) I_{\left[R^{+}\right]} & \text {if } & \mu_{m}\left(\vec{X}_{t}\right)>M_{m}\left(\vec{X}_{t}\right) \\
& =M_{m}\left(\vec{X}_{t}\right) I_{\left[R^{-}\right]}+\mu_{m}\left(\vec{X}_{t}\right) I_{\left[R^{+}\right]} & \text {if } & \mu_{m}\left(\vec{X}_{t}\right) \leqslant M_{m}\left(\vec{X}_{t}\right)
\end{array}
$$

where $R^{-}=\left\{\vec{X}_{t}: \mu_{m}\left(\vec{X}_{t}\right) \leqslant A R_{m}\left(\vec{X}_{t}\right)\right\}$ and $R^{+}=\left\{\vec{X}_{t}: \mu_{m}\left(\vec{X}_{t}\right)>A R_{m}\left(\vec{X}_{t}\right)\right\}$. If the true model is nonlinear, theoretically we can still find a reasonable approximate linear model. Then, the new forecast is the nonlinear MMSE forecast unless its relative forecast performance is worse than the MMAE forecast, which certainly includes the case when the MMSE performs worse than the linear forecast. In Example 2, the $M F_{m}\left(\vec{X}_{t}\right)$ forecast of model (2) is shown to be the MMSE forecast for $\vec{X}_{t}$ lying in a high stationary density region. In Theorem 7 , assuming the true model is nonlinear, $P_{m}\left(\vec{X}_{t}\right)$ of the forecast $M F_{m}\left(\vec{X}_{t}\right)$ is shown to be the maximum of those of MMSE and MMAE forecasts. 
To indicate the predictor used in the comparison probability, let $P_{m}\left(\vec{X}_{t}, \mu_{m}\right), P_{m}\left(\vec{X}_{t}, M_{m}\right)$ and $P_{m}\left(\vec{X}_{t}, M F_{m}\right)$ denote the $P_{m}\left(\vec{X}_{t}\right)$ of $\mu_{m}\left(\vec{X}_{t}\right), M_{m}\left(\vec{X}_{t}\right)$ and $M F_{m}\left(\vec{X}_{t}\right)$ respectively.

Theorem 7 If model (3) is the true model, then

$$
P_{m}\left(\vec{X}_{t}, M F_{m}\right)=\max \left\{P_{m}\left(\vec{X}_{t}, \mu_{m}\right), P_{m}\left(\vec{X}_{t}, M_{m}\right)\right\} \geqslant \frac{1}{2}
$$

Remark It seems that the computation of $M F_{m}$ is more complicated than computing $M_{m}\left(\vec{X}_{t}\right)$ for $M_{m}\left(\vec{X}_{t}\right)$ is involved in the definition of $M F_{m}$. However, in many cases, the relation between $\mu_{m}\left(\vec{X}_{t}\right)$ and $M_{m}\left(\vec{X}_{t}\right)$ can be determined before computing them (see Example 2 below). Thus, $M_{m}\left(\vec{X}_{t}\right)$ needs only be computed when $M F_{m}=M_{m}$.

Example 2 (Example 1, continued) The $m$-step conditional density of $X_{t+m}$ given $X_{t}$ for model (2) is

$$
f_{m}\left(x \mid X_{t}\right)=k_{1}^{(m)} \varphi\left(x-\operatorname{sign}\left(X_{t}\right) \alpha\right)+k_{2}^{(m)} \varphi\left(x+\operatorname{sign}\left(X_{t}\right) \alpha\right)
$$

where $\beta=1-2 \Phi(\alpha), k_{1}^{(m)}$ and $k_{2}^{(m)}$ are given in Corollary 4 . The skewness of $f_{m}\left(x \mid x_{t}\right)$ is affected by both $m$ and $\beta$ which determine the weights of the linear combination of $\varphi(x+\alpha)$ and $\varphi(x-\alpha)$. See Figures 12 and 13 for the plots of $f_{m}\left(x \mid x_{t} \leqslant 0\right)$ versus $x$ when $\alpha=1,2, m=1,2,3,4$, 5. It can be proved (see the Appendix) that

(1) $\mu_{m}\left(X_{t}\right) \leqslant M_{m}\left(X_{t}\right)$ if $X_{t} \leqslant 0$ and $m$ is odd or $X_{t}>0$ and $m$ is even

(2) $\mu_{m}\left(X_{t}\right)>M_{m}\left(X_{t}\right)$ if $X_{t} \leqslant 0$ and $m$ is even or $X_{t}>0$ and $m$ is odd.

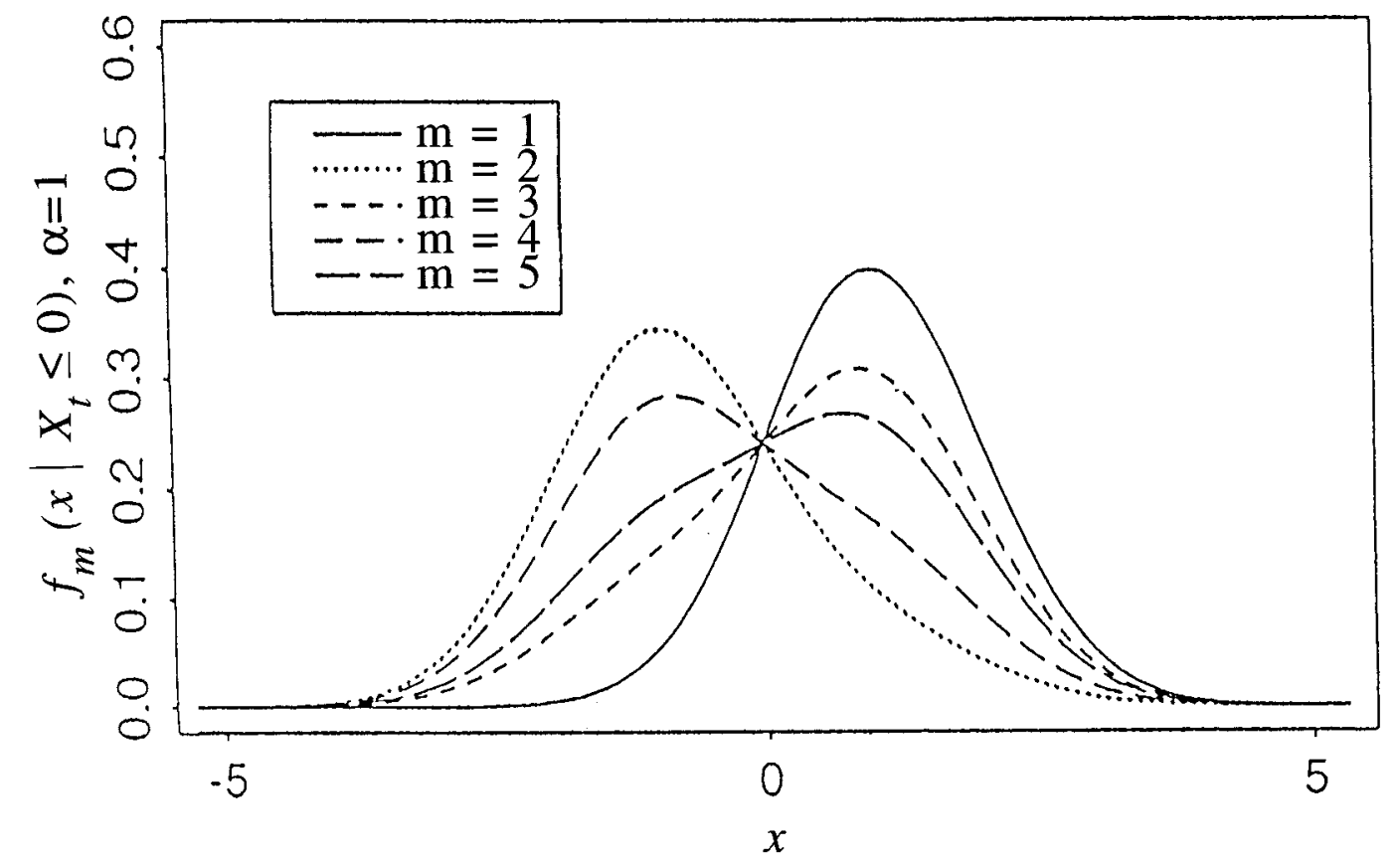

Figure 12 


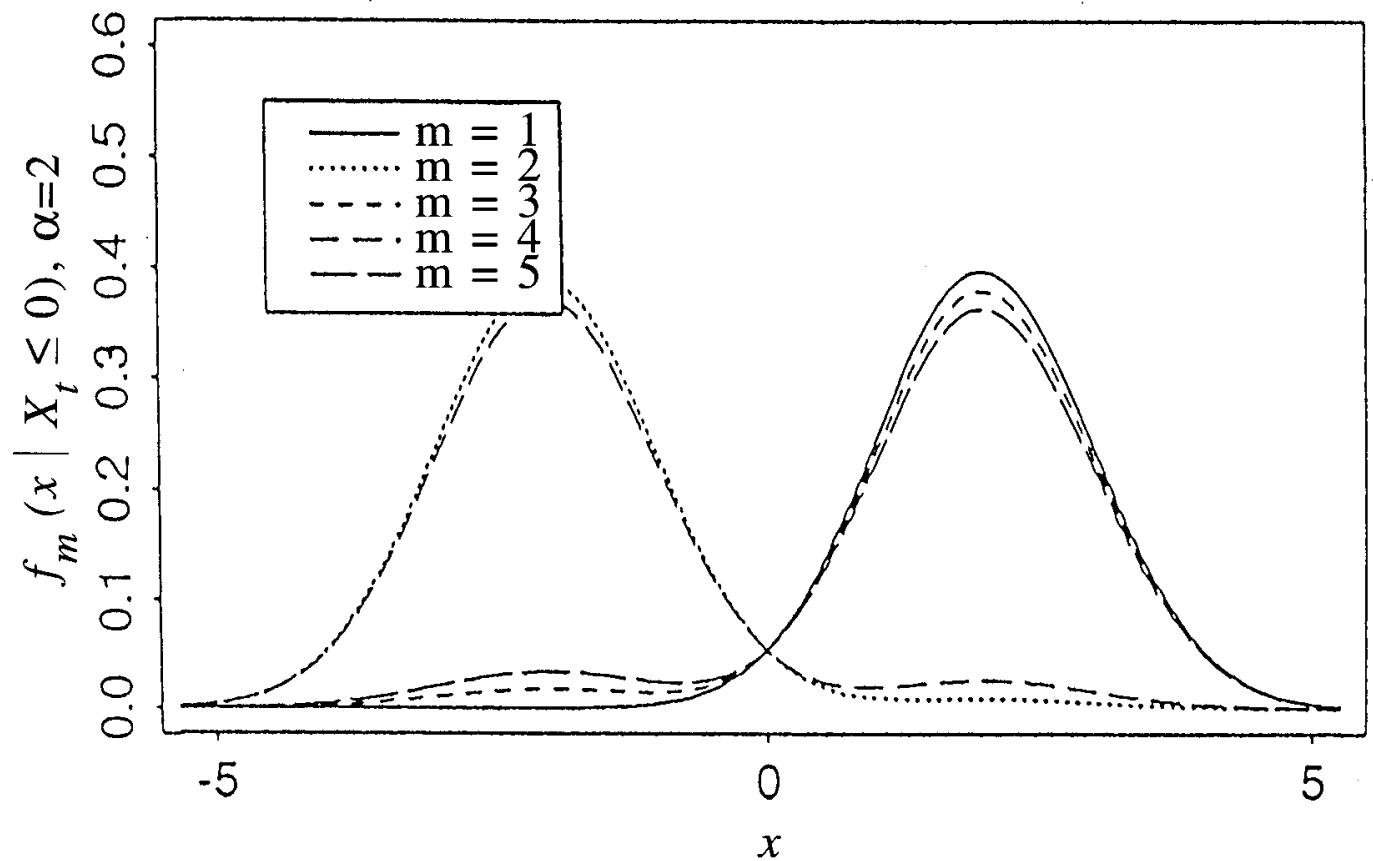

Figure 13

The new forecast of model (2) with $\alpha=1.5$ can be simplified as $M F_{m}\left(X_{t}\right)=\mu_{m}\left(X_{t}\right) I_{\left[K_{m}\right]}+$ $M_{m}\left(X_{t}\right) I_{\left[K_{m}^{c}\right]}, \quad$ where $\mu_{m}\left(X_{t}\right)=-\alpha \beta^{m-1} \operatorname{sign}\left(X_{t}\right), \quad K_{m}=\left\{X_{t}:\left|X_{t}\right| \leqslant\left|\alpha \beta^{m-1} / \hat{\phi}^{m}\right|\right\}, \quad K_{m}^{c}=$ $\left\{X_{t}:\left.\left|X_{t}\right|\right|^{m}\left|\alpha \beta^{m-1} / \hat{\phi}^{m}\right|\right\}$ and $\hat{\phi} \cong-0.7$. See Table I in Example 1 for the values of $\alpha \beta^{m-1} / \hat{\phi}^{m}$ for $m=1,2, \ldots, 10$. Therefore the proposed forecast $M F_{m}\left(X_{t}\right)$ is the $m$-step conditional mean for origins $X_{t}$ in high stationary probability density regions and is the $m$-step conditional median for origins $X_{t}$ in low stationary density regions. The plots of $P_{m}\left(X_{t}\right)$ versus $X_{t}$ for $M F_{m}\left(X_{t}\right), \mu_{m}\left(X_{t}\right)$ and $M_{m}\left(X_{t}\right), m=1,2,3$ are given in Figures 4-6.

\section{DISCUSSION AND CONCLUSIONS}

If the true model is completely known or partially known (or known functional form with unknown parameters) and the criterion is chosen as the RMSE (RMAE), then the best forecast is the conditional mean (median) forecast. However, if the comparison probability $P_{m}\left(\vec{X}_{t}\right)$ is chosen as the criterion, the case becomes complicated. In the theorems in the third section, the expressions of $P_{m}\left(\vec{X}_{t}\right)$ are given. It has been proven that $P_{m}\left(\vec{X}_{t}\right)$ is an increasing function of the forecast distance $\xi_{m}\left(\vec{X}_{t}\right)$ for origin $\vec{X}_{t}$ in disjoint regions. To determine which predictor should be used, one has to compute the two predictors in comparison. This is seemingly not a good deal because the purpose is to use the linear forecast to save computation time. However, there may be alternative ways to determine which predictor should be used. As illustrated in Example 2, when computing the $M F_{m}\left(\vec{X}_{t}\right)$, one can determine whether $M_{m}\left(\vec{X}_{t}\right)$ is needed. Also, as noted by 
Pemberton, when $\vec{X}_{t}$ is in the region of high density values, the distance $\xi_{m}\left(\bar{X}_{t}\right)$ generally (although not always) has smaller values and hence the linear and nonlinear forecasts are comparable.

The newly proposed forecast provides an alternative way of selecting conditional mean or conditional median forecast when the $m$-step conditional distribution is skewed. However, when the $m$-step conditional distribution is not of a location scale family or when the model is more complicated than the piecewise constant SETAR model the property of $P_{m}\left(\vec{X}_{t}\right)$ still needs further studies.

\section{APPENDIX}

Proof of Theorem 3 Since the conditional distribution of $X_{t+m}$ given $X_{t} \in\left(c_{j-1}, c_{j}\right]$ is $F_{m, j}(x)=$ $\sum_{i=1}^{l} k_{i j}^{(m)} \Phi\left(x-\alpha_{i}\right)$ (see Pemberton, 1990),

$$
\begin{aligned}
F_{m, j}\left(Q_{m}\left(X_{t}\right)\right)= & {\left[\sum_{i=1}^{l} k_{i j}^{(m)} \Phi\left(\frac{\xi_{m}\left(X_{t}\right)}{2}+\mu_{m}\left(X_{t}\right)-\alpha_{i}\right)\right] I_{\left[R^{-}\right]} } \\
& +\left[\sum_{i=1}^{l} k_{i j}^{(m)} \Phi\left(-\frac{\xi_{m}\left(X_{t}\right)}{2}+\mu_{m}\left(X_{t}\right)-\alpha_{i}\right)\right] I_{\left[R^{+}\right]}
\end{aligned}
$$

Given $X_{t} \in\left(c_{j-1}, c_{j}\right], \mu_{m}\left(X_{t}\right)=\sum_{i=1}^{l} k_{i j}^{(m)} \alpha_{i}$. Therefore, the result is obtained by setting $\alpha_{i j}^{(m)}=\mu_{m}\left(X_{t}\right)-\alpha_{i}$ and by model (5). QED

Proof of Corollary 4 The conditional distribution of $X_{t+m}$ given $X_{t}$ is

$$
F_{m}(x)=k_{2}^{(m)} \Phi\left(x+\operatorname{sign}\left(X_{t}\right) \alpha\right)+k_{1}^{(m)} \Phi\left(x-\operatorname{sign}\left(X_{t}\right) \alpha\right)
$$

Therefore, by model (5), for $X_{t}$ in $R^{-}$

$$
\begin{aligned}
P_{m}\left(X_{t}\right)= & F_{m}\left(Q_{m}\left(X_{t}\right)\right) \\
= & k_{2}^{(m)} \Phi\left(Q_{m}\left(X_{t}\right)+\operatorname{sign}\left(X_{t}\right) \alpha\right)+k_{1}^{(m)} \Phi\left(Q_{m}\left(X_{t}\right)-\operatorname{sign}\left(X_{t}\right) \alpha\right) \\
= & k_{2}^{(m)} \Phi\left(\frac{1}{2} \xi_{m}\left(X_{t}\right)-\alpha \beta^{m-1} \operatorname{sign}\left(X_{t}\right)+\operatorname{sign}\left(X_{t}\right) \alpha\right) \\
& +k_{1}^{(m)} \Phi\left(\frac{1}{2} \xi_{m}\left(X_{t}\right)-\alpha \beta^{m-1} \operatorname{sign}\left(X_{t}\right)-\operatorname{sign}\left(X_{t}\right) \alpha\right) \\
= & k_{2}^{(m)} \Phi\left(\frac{1}{2} \xi_{m}\left(X_{t}\right)+2 \alpha \operatorname{sign}\left(X_{t}\right) k_{1}^{(m)}\right)+k_{1}^{(m)} \Phi\left(\frac{1}{2} \xi_{m}\left(X_{t}\right)-2 \alpha \operatorname{sign}\left(X_{t}\right) k_{2}^{(m)}\right) \\
= & H_{m}\left(\frac{1}{2} \xi_{m}\left(X_{t}\right)\right)
\end{aligned}
$$

Similarly, one can prove for $X_{t}$ in $R^{+}$

$$
P_{m}\left(X_{t}\right)=1-F_{m}\left(Q_{m}\left(X_{t}\right)\right)=1-H_{m}\left(-\frac{1}{2} \xi_{m}\left(X_{t}\right)\right)
$$

Proof of Theorem 5 Since

$$
E\left[\left(X_{t+m}-A R_{m}\left(\vec{X}_{t}\right)\right)^{2} \mid \vec{X}_{t}\right]=\operatorname{MSE}\left(\mu_{m}\left(\vec{X}_{t}\right) \mid \vec{X}_{t}\right)+\left(A R_{m}\left(\vec{X}_{t}\right)-\mu_{m}\left(\vec{X}_{t}\right)\right)^{2}
$$


it is easy to derive model (8). Furthermore, since

$$
\begin{aligned}
E|x-a|-E|x-b| & =(b-a)(1-2 F(a))-2 \int_{a}^{b}(b-x) \mathrm{d} F(x), \quad \text { if } \quad b>a \\
& =(a-b)(2 F(a)-1)-2 \int_{b}^{a}(x-b) \mathrm{d} F(x), \quad \text { if } \quad b<a
\end{aligned}
$$

thus model (9) is derived by letting $a=M_{m}\left(\vec{X}_{t}\right)$ and $b=A R_{m}\left(\vec{X}_{t}\right)$.

Proof of Theorem 7 First consider the case when $\mu_{m}\left(\vec{X}_{t}\right)>M_{m}\left(\vec{X}_{t}\right)$.

(1) If $A R_{m}\left(\vec{X}_{t}\right) \geqslant \mu_{m}\left(\vec{X}_{t}\right)$, then $M F_{m}\left(\vec{X}_{t}\right)=\mu_{m}\left(\vec{X}_{t}\right)$ and

$$
\begin{aligned}
P_{m}\left(\vec{X}_{t}, M F_{m}\right)= & F_{m}\left(Q_{m}\left(\mu_{m}\right) \mid \vec{X}_{t}\right) \\
& >F_{m}\left(Q_{m}\left(M_{m}\right) \mid \vec{X}_{t}\right)\left(\geqslant \frac{1}{2}\right) \\
= & P_{m}\left(\vec{X}_{t}, M_{m}\right)
\end{aligned}
$$

where $F_{m}\left(\cdot \mid \vec{X}_{t}\right)$ is the conditional distribution of $X_{t+m}$ given $\vec{X}_{t}, Q_{m}\left(\mu_{m}\right)=\frac{1}{2}\left(\mu_{m}\left(\vec{X}_{t}\right)+A R_{m}\left(\vec{X}_{t}\right)\right)$ and $Q_{m}\left(M_{m}\right)=\frac{1}{2}\left(M_{m}\left(\vec{X}_{t}\right)+A R_{m}\left(\vec{X}_{t}\right)\right)$.

(2) If $M_{m}\left(\vec{X}_{t}\right) \leqslant A R_{m}\left(\vec{X}_{t}\right)<\mu_{m}\left(\vec{X}_{t}\right)$, then $M F_{m}\left(\vec{X}_{t}\right)=M_{m}\left(\vec{X}_{t}\right)$ and

$$
\begin{aligned}
P_{m}\left(\vec{X}_{t}, M F_{m}\right)= & F_{m}\left(Q_{m}\left(M_{m}\right) \mid \vec{X}_{t}\right) \\
& \geqslant F_{m}\left(M_{m}\left(X_{t}\right) \mid \vec{X}_{t}\right)=1-F_{m}\left(M_{m}\left(X_{t}\right) \mid \vec{X}_{t}\right)\left(=\frac{1}{2}\right) \\
& >1-F_{m}\left(Q_{m}\left(\mu_{m}\right) \mid \vec{X}_{t}\right)=P_{m}\left(\vec{X}_{t}, \mu_{m}\right)
\end{aligned}
$$

(3) If $A R_{m}\left(\vec{X}_{t}\right)<M_{m}\left(\vec{X}_{t}\right)$, then $M F_{m}\left(\vec{X}_{t}\right)=M_{m}\left(\vec{X}_{t}\right)$ and

$$
\begin{aligned}
P_{m}\left(\vec{X}_{t}, M F_{m}\right)= & 1-F_{m}\left(Q_{m}\left(M_{m}\right) \mid \vec{X}_{t}\right)\left(\geqslant \frac{1}{2}\right) \\
& >1-F_{m}\left(Q_{m}\left(\mu_{m}\right) \mid \vec{X}_{t}\right) \\
= & P_{m}\left(\vec{X}_{t}, \mu_{m}\right)
\end{aligned}
$$

When $\mu_{m}\left(\vec{X}_{t}\right) \leqslant M_{m}\left(\vec{X}_{t}\right)$, the result can be obtained similarly. QED

Proof of (1) and (2) in Example 2 Since $\varphi(x)$ is decreasing for $x \geqslant 0$,

$$
\frac{1}{k} \int_{0}^{2 \alpha k} \varphi(x) \mathrm{d} x=\frac{1}{k}\left(\Phi(2 \alpha k)-\frac{1}{2}\right)
$$

is decreasing in $k$. First consider the case for $X_{t} \leqslant 0$. Let $F_{m}\left(\cdot \mid X_{t}\right)$ denote the conditional distribution of $X_{t+m}$ given $X_{t}$, then

$$
\begin{aligned}
F_{m}\left(\mu_{m}\left(X_{t}\right) \mid X_{t}\right)-\frac{1}{2}= & k_{1}^{(m)} \Phi\left(2 \alpha k_{2}^{(m)}\right)+k_{2}^{(m)} \Phi\left(-2 \alpha k_{1}^{(m)}\right)-\frac{1}{2} \\
= & k_{1}^{(m)} k_{2}^{(m)}\left[\frac{1}{k_{2}^{(m)}}\left(\Phi\left(2 \alpha k_{2}^{(m)}\right)-\frac{1}{2}\right)-\frac{1}{k_{1}^{(m)}}\left(\Phi\left(2 \alpha k_{1}^{(m)}\right)-\frac{1}{2}\right)\right] \\
& \geqslant 0 \quad \text { if and only if } k_{2}^{(m)} \leqslant k_{1}^{(m)}
\end{aligned}
$$

(C) 1997 John Wiley \& Sons, Ltd. 
Thus, $\mu_{m}\left(X_{t}\right) \geqslant M_{m}\left(X_{t}\right) \Leftrightarrow F_{m}\left(\mu_{m}\left(X_{t}\right) \mid X_{t}\right) \geqslant \frac{1}{2} \Leftrightarrow k_{2}^{(m)} \leqslant k_{1}^{(m)} \Leftrightarrow m$ is even, since $\beta<0$.

The case when $X_{t}>0$ can be similarly proved. QED

\section{ACKNOWLEDGEMENTS}

The authors are grateful to Zhidong Bai for his helpful comments and suggestions. The work presented in this paper was supported by the National Science Council of the Republic of China, Grant No. NSC 82-0208-N-110-064.

\section{REFERENCES}

Davies, N., Petruccelli, J. D. and Pemberton, J., 'An automatic procedure for identification, estimation and forecasting self exciting threshold autoregressive models', Statistician, 37 (1988), 199-204.

Oster, G. and Ipaktchi, A., 'Population cycles', Theor. Chem. Periodicity, in Eyring, H. and Henderson, D. (eds), Chem \& Biol, New York: Academic Press, 111-32.

Ozaki, T., 'Nonlinear time series models and dynamical systems', in Hannan, E. J., Krishnaiah, P. R. and Rao, M. M. (eds), Handbook of Statistics 5, Amsterdam: North-Holland, 1985, $25-83$.

Pemberton, J., 'Forecast accuracy of nonlinear time series models', Tech. Rep. 77, Statistics Research Center, Graduate School of Business, University of Chicago, 1989.

Pemberton, J., 'Piecewise constant autoregressive models for univariate time series', Tech. Rep. MCS-90-04, Department of Mathematics \& Computer Science, University of Salford, 1990.

Priestly, M. B., Spectral Analysis and Time Series, New York: Academic Press, 1989.

Tiao, G. C. and Tsay, R. S., 'Some advances in nonlinear and adaptive modeling in time series', J. Forecast., 13 (1994), 109-31.

Tong, H., Non-linear Time Series: A dynamical systems approach, Oxford: Oxford University Press, 1990.

Authors' address:

Meihui Guo and Y. K. Tseng, Department of Applied Mathematics, National Sun Yat-sen University, Kaohsiung, ROC 80424. 